УДК 616-039.57-036.88-07

DOI 10.17816/pmi35168-74

РЕТРОСПЕКТИВНЫЙ АНАЛИЗ СЛУЧАЕВ СМЕРТИ

НА ДОМУ ПАЦИЕНТОВ, НАХОДИВШИХСЯ

ПОД ДИСПАНСЕРНЫМ НАБЛЮДЕНИЕМ

Я.П. Сандаков

Диагностический центр № 3 Департамента здравоохранения г. Москвы, Россия

\title{
RETROSPECTIVE ANALYSIS OF EVENTS OF DEATH AT HOME AMONG PATIENTS UNDER FOLLOW-UP OBSERVATION
}

\author{
Ya.P. Sandakov \\ Diagnostic Center № 3 of Moscow Healthcare Department, Russian Federation
}

Цель. Изучить особенности обращения за медицинской помощью пациентов, находившихся под диспансерным наблюдением, из числа умерших на дому.

Материалы и методы. С применением методов описательной и индуктивной статистики выполнен анализ сведений, полученных из медицинских карт амбулаторного больного, 100 пациентов, находившихся на диспансерном наблюдении, из числа умерших на дому.

Результаты. Средний возраст смерти составил 74,9 \pm 13,4 г., мужчины - 65,8 \pm 14,1 г., женщины 79,2 \pm 10,9 г. В 78 \% случаев причиной диспансерного наблюдения и в 85 \% причиной смерти были болезни сердечно-сосудистой системы. У 30 \% пациентов заболевание было выявлено при диспансеризации. Средняя продолжительность болезни к моменту смерти - 13,0 \pm 8,0 г., у пенсионеров - 13,7 \pm 8,5 г., у трудоспособных $-9,6 \pm$ 4,3 г. Средняя длительность диспансерного наблюдения составила 9,2 \pm 5,8 г. Среднее число плановых посещений равнялось $2,8 \pm 0,89$, а фактических посещений за последний год диспансерного наблюдения - 2,4 \pm 1,2. Инвалидность имелась у 77,6 \% человек, сопутствующие заболевания у всех пациентов. За последний год были зафиксированы обострения у $92 \%$ пациентов, вызовы скорой и неотложной помощи у 80 \%, случаи госпитализации - 52 \%, из них 55,8 \% - экстренные.

Выводы. Отсутствие в изучаемой группе значимых разницы в продолжительности болезни между пенсионерами и трудоспособными ( $m=0,16, p=1,43)$, а также корреляции возраста и продолжительности болезни $\left(\chi^{2}=0,19, p=0,2\right)$ косвенно свидетельствует о влиянии продолжительность болезни на ее исход, а не возраста. Посещаемость, характеризующая клиническую приверженность лечению, не зависит от возраста $\left(\chi^{2}=-0,19, p=0,18\right)$, пола $(m=1,0, p=0,32)$, пути выявления заболевания $(f=0,9$, $p=0,4)$, класса основного заболевания $(f=0,91, p=0,44)$, продолжительности заболевания $\left(\chi^{2}=-0,13\right.$, $p=0,49)$, обострений $\left(\chi^{2}=-0,17, p=0,24\right)$, сопутствующих заболеваний $\left(\chi^{2}=0,006, p=0,9\right)$. Число вызовов скорой и неотложной помощи, госпитализаций, экстренных госпитализаций не зависит от длительности диспансерного наблюдения, регулярности и числа фактических посещений врача $(p>0,05)$, что указывает на неудовлетворительное качество диспансерного наблюдения.

ключевые слова. Диспансерное наблюдение, смерть на дому.

(ㄷ) Сандаков Я.П., 2018

тел. +7 (495) 9191175

e-mail: Sand_2004@mail.ru

[Сандаков Я.П. - заместитель главного врача по медицинской части]. 
Aim. To study the peculiarities of asking for medical care by patients from among those dead at home, who had been under follow-up observation.

Materials and methods. The data, received from ambulatory medical records of 100 dead at home patients with follow-up, were analyzed using the methods of descriptive and inductive statistics.

Results. A mean age of death was $74.9 \pm 13.4$ years, men $-65.8 \pm 14.1$ years, women $-79.2 \pm 10.9$ years. Cardiovascular diseases were the cause of follow-up observation in $78 \%$ of cases and the cause of death in $85 \%$; in $30 \%$ of patients the disease was detected while carrying out prophylactic medical examination. A mean duration of disease by the moment of death was $13.0 \pm 8.0$ years, among pensioners $13.7 \pm 8.5$ years, in able-bodied persons $9.6 \pm 4.3$ years. A mean duration of follow-up observation was $9.2 \pm 5.8$ years. An average number of planned visits was $2.8 \pm 0.89$, but the number of real visits during the last year of follow-up observation was $2.4 \pm 1.2$. Disability was registered in $77.6 \%$ of patients, concomitant diseases - in all patients. For the last year, exacerbations were recorded in $92 \%$ of patients, emergency calls - in $80 \%$, hospitalization events - $52 \%$, including $55.8 \%$ - urgent ones.

Conclusions. The absence of significant difference regarding the duration of disease between pensioners and able-bodied patients $(m=0.16, p=1.43)$ as well as the absence of correlation between the age and duration of disease $\left(\chi^{2}=0.19, p=0.2\right)$ indirectly prove the influence of duration of disease on its outcome, but not the age. Attendance, characterizing clinical loyalty to treatment, does not depend on age $\left(\chi^{2}=-0.19\right.$, $p=0.18)$, sex $(m=1.0, p=0.32)$, way of detecting disease $(f=0.9, p=0.4)$, class of main disease $(f=0.91$, $p=0.44)$, duration of disease $\left(\chi^{2}=-0.13, p=0.49\right)$, exacerbations $\left(\chi^{2}=-0.17, p=0.24\right)$, concomitant diseases $\left(\chi^{2}=0.006, p=0.9\right)$. The number of emergency calls, hospitalizations, urgent hospitalizations does not depend on duration of follow-up observation, regularity and number of real visits to a doctor $(p>0.05)$ that indicates unsatisfactory quality of follow-up observation.

Key words. Follow-up observation, death at home.

\section{ВВЕДЕНИЕ}

На фоне увеличения нормативов финансовых затрат медицинской помощи в амбулаторных условиях, установленных Программой государственных гарантий оказания гражданам Российской Федерации бесплатной медицинской помощи, активнее выдвигаются требования к эффективности первичной медико-санитарной помощи $[1,5,7]$.

В то же время при изучении диспансерного наблюдения внимание исследователей чаще сфокусировано на охвате диспансерным наблюдением, удельном весе диспансерных посещений, сроках постановки на учет, уровне удовлетворенности населения, своевременности обследования $[2,4,6]$. Но не стоит забывать, что критерием результативности диспансерного наблюдения является частота вызовов скорой помощи, госпитализаций, в том числе экстренных [3]. Кроме того, важность диспансерного наблю- дения заключается в формировании клинической приверженности лечению, которая является залогом успеха медицинской помощи [8]. Известно, что низкая приверженность пациентов ведет к увеличению расходов на здравоохранение и отрицательным результатам в отношении здоровья [9]. В этой связи иелью настоящего исследования стало изучение особенностей обращений за медицинской помощью пациентов, находившихся под диспансерным наблюдением, из числа умерших на дому.

\section{МАТЕРИАЛЫ И МЕТОДЫ ИССЛЕДОВАНИЯ}

Выполнена выкопировка данных из 100 медицинских карт амбулаторного больного пациентов, находившихся на диспансерном наблюдении и умерших на дому в 2016-2017 гг. Для анализа применялись методы описательной статистики: расчет средних, стандартных отклонений, степеней сво- 
боды (df), 95\%-ных доверительных интервалов (ДИ95\%), числа наблюдений, частот, долей. Оценка равенств, регрессии, дисперсии, корреляции проводилась с применением методов индуктивной статистики. Неравенство средних для двух независимых или парных выборок, а также оценка коэффициентов однофакторной линейной регрессии подтверждались расчетом критерия Стьюдента $(t)$, когда двухсторонняя значимость $(p)$ была меньше 0,05. Достоверность различий между процентными долями двух выборок подтверждались расчетом критерия Фишера, если эмпирическое значение $\varphi$ было выше критического для $p=0,05$. Сравнение более двух независимых выборок проводилось методом однофакторного дисперсионного анализа $f$, разница подтверждалась при значимости $(p)$ меньше 0,05. Парная корреляция подтверждалась расчетом критерия Пирсона $\left(\chi^{2}\right)$, когда двухсторонняя значимость $(p)$ была меныше 0,05. Статистическая обработка данных проводилась с использованием программного продукта IBM SPSS Statistics, версия 23.

\section{РЕЗУЛЬТАТЫ И ИХ ОБСУЖДЕНИЕ}

Средний возраст смерти составил 74,9 \pm \pm 13,4 г., мужчины - 65,8 \pm 14,1 г., женщины $79,2 \pm 10,9$ г. Женщины умирают в более позднем возрасте $(t=3,3, p=0,02, d f=98$, ДИ95\% (4,7; 19,1). В 16 \% случаев смерть наступила у лиц в трудоспособном возрасте, средний возраст пациентов трудоспособной группы - 51,4 \pm 5,9 г. Хотя среди умерших больше женщин (62 \%), по сравнению с мужчинами (38 \%), доля мужчин, умерших в трудоспособном возрасте (36,8 \% от общего числа умерших мужчин), выше, чем доля женщин, умерших в трудоспособном возрасте (3,2 \% от общего числа умерших женщин). Средний возраст смерти лиц трудоспособно- го возраста - 51,4 \pm 5,9 г. (женщины 51,5 \pm 0,7 г., мужчины - 50,6 \pm 5,9 г.). Разница в возрасте между трудоспособными мужчинами и женщинами статистически незначима $(t=2,2, p=0,8, d f=14$, ДИ $95 \%-(8,3 ; 10,1))$.

В 78 \% случаев причиной диспансерного наблюдения были болезни сердечнососудистой системы, 12 \% - болезни эндокринной системы, 8 \% - болезни органов пищеварения, 2 \% - болезни органов дыхания. Причиной смерти лиц, находящихся на диспансерном наблюдении, стали в 85 \% случаев болезни сердечно-сосудистой системы, в 8,8 \% - новообразования, 5,9 \% - болезни эндокринной системы. Самой частой причиной диспансерного наблюдения (38 \%) и причиной смерти (29,4%) была атеросклеротическая болезнь сердца I25.1. При этом 90 \% пациентов находилось под наблюдением врача-терапевта, а 10 \% - эндокринолога. Редкое привлечение узких профильных специалистов к диспансерному наблюдению является неоправданным, на наш взгляд, что подтверждают нижеприведенные сведения. Протоколы изучения летального исхода были вклеены в медицинскую карту амбулаторного больного в 68 \% случаев. В 8,8 \% случаев среди зафиксированных в медицинской документации диагнозов отсутствовали состояния, связанные с причиной наступившей смерти. Например, среди сопутствующих и основного заболеваний указаны коды МКБ-10 N49, I11.9, I67.2, I25.1, а причина смерти С16.8 «поражение желудка, выходящее за пределы одной вышеуказанной локализации и более» или основное и сопутствующие заболевания I 25.1, I 67.2, I 11.9, I 67.8, а причина смерти E11.0 «сахарный диабет 2-го типа, кома». Причем срок диспансерного наблюдения в среднем по данным случаям составил $9 \pm 0,8$ г., а средняя частота посещения $2 \pm 0,8$ раза в год. 
Средняя продолжительность болезни (срок с момента первичного установления диагноза) в изучаемой группе $13,0 \pm 8,0$ г., у пенсионеров - 13,7 $\pm 8,5$ г., у трудоспособных $-9,6 \pm$ 4,3 г., у мужчин $-13,4 \pm 10,4$ г., женщин - 12,8 \pm 6,8 г. Разница в продолжительности болезни статистически не значима между трудоспособными и пенсионерами $(m=1,43, p=0,16)$, между мужчинами и женщинами $(m=0,28, p=0,79)$. Также не обнаружено статистически значимой корреляции возраста и продолжительности болезни $\left(\chi^{2}=0,19, p=0,2\right)$. Этот факт косвенно свидетельствует, что продолжительность болезни (а не возраст пациента) оказывает влияние на исход болезни.

У 68 \% пациентов диагноз установлен впервые, у 32 \% - повторно. Заболевание выявлено при: диспансеризации - у 30 \% пациентов, при профилактическом осмотре у 22 \%, ином обращении за оказанием медицинской помощи - у 36 \%, в другой медицинской организации - у $12 \%$.

Средняя длительность диспансерного наблюдения составила 9,2 \pm 5,8 г. Средний срок диспансерного наблюдения среди трудоспособных лиц 8,2 \pm 4,7 г, пенсионеров - 9,4 \pm 6,1 г. $(p=0,6)$, среди мужчин - 11,5 $\pm 8,4$, женщин $8,1 \pm 3,7$ ( $p=0,055)$. Отсутствие статистически значимой разницы между продолжительностью болезни и длительностью диспансерного наблюдения свидетельствует о позднем взятии пациентов под наблюдение ( $m=5,6, p<0,01)$.

Среднее число плановых посещений равнялось 2,8 $\pm 0,89$, а фактических посещений за последний год диспансерного наблюдения составило $2,4 \pm 1,2$. Статистически значимая разница между плановыми и фактическими посещениями $(m=2,08, p=0,04)$ указывает на низкую клиническую приверженность лечению. Половина пациентов фактически посещали врача в соответствии с плановыми посещениями, 38 \% - реже, $12 \%$ - чаще. Интересно, что в группе с двумя плановыми посещениями частота фактических посещений была статистически значимо выше ( $\varphi=5,3$ при $p<0,01)$, а пропусков запланированных посещений - ниже ( $\varphi=5,4$ при $p<0,01)$, чем в группе, где было установлено 3 плановых посещения (таблица).

\section{Распределение лиц по типу фактических посещений}

\begin{tabular}{|l|c|c|c|}
\hline \multirow{2}{*}{$\begin{array}{c}\text { Число пла- } \\
\text { новых } \\
\text { посещений } \\
\text { в год }\end{array}$} & \multicolumn{3}{|c|}{$\begin{array}{c}\text { Лица, \%, у которых число } \\
\text { фактических посещений }\end{array}$} \\
\cline { 2 - 4 } & $\begin{array}{c}\text { меньше } \\
\text { плановых }\end{array}$ & $\begin{array}{c}\text { равно } \\
\text { плановым }\end{array}$ & $\begin{array}{c}\text { больше } \\
\text { плановых }\end{array}$ \\
\hline 2 & 7,4 & 81,5 & 11,1 \\
\hline 3 и более & 73,9 & 13,1 & 13,0 \\
\hline
\end{tabular}

Не выявлено зависимости фактических посещений от возраста $\left(\chi^{2}=-0,19, p=0,18\right)$, пола ( $m=1,0, p=0,32)$, пути выявления заболевания $(f=0,9, p=0,4)$, продолжительности болезни $\left(\chi^{2}=-0,13, p=0,49\right)$, длительности диспансерного наблюдения $\left(\chi^{2}=-0,1\right.$, $p=0,36)$, класса основного заболевания $(f=0,91, p=0,44)$.

У 92 \% пациентов были зафиксированы обострения состояний. Среднее число обострений равнялось $3,2 \pm 2,2$. Зависимости обострений от возраста $\left(\chi^{2}=0,07, p=0,65\right)$, продолжительности болезни $\left(\chi^{2}=0,24\right.$, $p=0,1)$, длительности диспансерного наблюдения $\left(\chi^{2}=0,26, p=0,07\right)$, числа $\left(\chi^{2}=-0,17, p=0,24\right)$ и регулярности $(f=1,4$, $p=0,3)$ фактических посещений, класса заболевания $(f=2,03, p=0,12)$ нет. Однако обнаружено, что частота обострений статистически значимо выше ( $m=2,55, p=0,014)$ у мужчин $(4,38 \pm 2,6)$, чем у женщин $(2,74 \pm 1,89)$.

Сопутствующие заболевания были зафиксированы у всех пациентов рассматри- 
ваемой группы (100 \%), в среднем на одного пациента приходилось 2,8 \pm 1,6 сопутствующего заболевания. Не обнаружено зависимости сопутствующих заболеваний от возраста $\left(\chi^{2}=-0,14, p=0,32\right)$, продолжительности болезни $\left(\chi^{2}=0,06, p=0,09\right)$, длительности диспансерного наблюдения $\left(\chi^{2}=0,09\right.$, $p=0,5)$, регулярности $(f=1,95, p=0,15)$ и числа $\left(\chi^{2}=0,006, p=0,9\right)$ фактических посещений. Обнаружены статистически значимые средняя прямая корреляция сопутствующих заболеваний и обострений $\left(\chi^{2}=0,43\right.$, $p=0,01)$, а также превышение $(m=2,8$, $p=0,008)$ частоты сопутствующих заболеваний у мужчин $(3,63 \pm 1,2)$ по сравнению с женщинами $(2,38 \pm 1,6)$. Эти сведения объясняют ранее упомянутое превышение частоты обострений у мужчин.

Вызовы скорой и неотложной помощи были отмечены у 80 \% пациентов на последнем году диспансерного наблюдения, в среднем 0,8 \pm 0,63 вызова. Не выявлена зависимость вызовов скорой и неотложной помощи от возраста $\left(\chi^{2}=-0,03, p=0,85\right)$, пола $(m=-1,23, p=0,23)$, заболевания $(f=1,42$, $p=0,25)$, продолжительности болезни $\left(\chi^{2}=-0,08, p=0,58\right)$, длительности диспансерного наблюдения $\left(\chi^{2}=-0,03, p=0,83\right)$, регулярности $(f=1,86, p=0,17)$ и числа фактических посещений $\left(\chi^{2}=-0,22, p=0,12\right)$, обострений $\left(\chi^{2}=-0,23, p=0,12\right)$, сопутствующих заболеваний $\left(\chi^{2}=-0,14, p=0,35\right)$.

За последний год диспансерного наблюдения у 52 \% лиц были зафиксированы случаи госпитализации, 16 \% были госпитализированы по причине основного заболевания, 50 \% - сопутствующего. В среднем по основному заболеванию было 0,2 \pm 0,5 случая госпитализаций на 1 пациента, по сопутствующим - 0,66 $\pm 0,77$. Среднее число госпитализаций по основному заболеванию у мужчин $(0,56 \pm 0,81)$ статистически значи- мо $(m=3,35, p=0,002)$ выше, чем у женщин $(0,06 \pm 0,24)$. Нет зависимости частоты госпитализаций по основному заболеванию от возраста $\left(\chi^{2}=-0,18, p=0,23\right)$, заболевания $(f=1,44, p=0,24)$, продолжительности болезни $\left(\chi^{2}=0,09, p=0,56\right)$, длительности диспансерного наблюдения $\left(\chi^{2}=-0,19, p=0,2\right)$, регулярности $(f=2,05, p=0,14)$ и числа фактических посещений $\left(\chi^{2}=0,03, p=0,86\right)$, обострений $\left(\chi^{2}=0,15, p=0,29\right)$, вызОвов скорой и неотложной помощи $\left(\chi^{2}=0,15, p=0,31\right)$.

В отношении госпитализации по сопутствующему заболеванию не обнаружена зависимость ее частоты от возраста $\left(\chi^{2}=0,07\right.$, $p=0,61)$, продолжительности болезни $\left(\chi^{2}=0,08, p=0,58\right)$, длительности диспансерного наблюдения $\left(\chi^{2}=0,19, p=0,19\right)$, peгулярности $(f=0,66, p=0,52)$ и числа фактических посещений $\left(\chi^{2}=-0,07, p=0,66\right)$, сопутствующих заболеваний $\left(\chi^{2}=-0,004\right.$, $p=0,98)$, вызовов скорой и неотложной помощи $\left(\chi^{2}=-0,09, p=0,55\right)$. Среднее число госпитализаций по сопутствующим заболеваниям статистически значимо ( $m=2,05$, $p=0,03)$ выше у мужчин $(1,06 \pm 1,1)$, чем у женщин $(0,53 \pm 0,7)$. Есть статистически значимая корреляция госпитализаций по сопутствующим заболеваниям с обострениями $\left(\chi^{2}=0,32, p=0,03\right)$. Высока доля экстренных госпитализаций - 55,8 \% от общего числа случаев госпитализаций и 80 \% от числа госпитализаций по основному заболеванию. Частота экстренных госпитализаций по основному заболеванию статистически значимо выше частоты экстренных госпитализаций по сопутствующим заболеваниям $(\varphi=4,78, p<0,01)$.

Для понимания обнаруженной статистически значимой разницы частоты сопутствующих заболеваний $(f=3,8, p=0,02)$ и госпитализаций по ним $(f=6,72, p=0,001)$ при различных классах основных заболеваниях, а также 
зависимости частоты госпитализаций по основному заболеванию от числа сопутствующих заболеваний $\left(\chi^{2}=0,34, p=0,014\right)$ требуются дополнительные исследования.

у 77,6 \% пациентов имелась инвалидность. Среди инвалидов больше лиц со 2-й группой (76,9 \%), с 1-й группой - 15,4\%. Частота инвалидности среди мужчин и женщин статистически одинакова $\varphi^{*} \ni$ мп $=1,49$, $p>0,05$. Не обнаружено статистически значимой разницы между инвалидами и лицами без инвалидности в возрасте ( $m=-1,9$, $p=0,06)$, продолжительностью болезни $(m=-1,2, p=0,24)$, длительностью диспансерного наблюдения ( $m=-0,77, p=0,44)$, числом плановых $(m=-0,97, p=0,34)$ и фактических ( $m=-0,14, p=0,89)$ посещений, сопутствующих заболеваний $(m=-0,87$, $p=0,39)$, обострений $(m=0,55, p=0,58)$, вызовов скорой и неотложной помощи $(m=-0,29, p=0,77)$, госпитализаций по основному заболеванию $(m=-0,29, p=0,77)$, экстренных госпитализаций по основному и сопутствующим заболеваниям ( $m=1,45$, $p=0,16)$. Но у лиц с инвалидностью статистически значимо $(m=2,5, p=0,02)$ ниже среднее число госпитализаций по сопутствующим заболеваниям, чем у лиц без инвалидности. Также среди лиц с разными группами инвалидности есть статистически значимая разница по среднему числу обострений $(f=3,4, p=0,04)$ : 1-я группа - 1,5 \pm 1,52 обострения; 2-я группа 3,5 \pm 1,8; 3-я группа - 3,0 $\pm 1,0$.

\section{Выводы}

1. Мужчины, особенно трудоспособного возраста, являются группой риска по развитию и неблагоприятному исходу неинфекционных хронических заболеваний: доля мужчин, умерших в трудоспособном возрасте, выше, чем доля женщин, умерших в тру- доспособном возрасте; у мужчин чаще зафиксированы обострения, сопутствующие заболевания, госпитализации по основному и сопутствующим заболеваниям $(p<0,05)$.

2. Качество диспансерного наблюдения неудовлетворительное: продолжительность болезни превышает средний срок диспансерного наблюдения $(m=5,6, p<0,01)$, высокая доля (80 \%) экстренных госпитализаций по основному заболеванию, разница между плановыми и фактическими посещениями $(m=2,08, p=0,04)$, посещаемость не зависят от длительности диспансерного наблюдения $\left(\chi^{2}=-0,1, p=0,36\right)$, число вызовов скорой и неотложной помощи, госпитализаций, экстренных госпитализаций не зависит от длительности диспансерного наблюдения, регулярности и числа фактических посещений врача $(\not>0,05)$; отметим редкое привлечение профильных специалистов к диспансерному наблюдению (причиной диспансерного наблюдения в 78 \% случаев и причиной смерти в $85 \%$ являются болезни сердечно-сосудистой системы, а 90 \% пациентов находилось под наблюдением врача-терапевта).

3. Отсутствие статистически значимой разницы в продолжительности болезни между пенсионерами и трудоспособными ( $m=0,16$, $p=1,43)$ в группе умерших, а также статистически значимой корреляции возраста и продолжительности болезни $\left(\chi^{2}=0,19, p=0,2\right)$ косвенно свидетельствует о влиянии продолжительность болезни на ее исход, а не возраста.

4. Посещаемость, характеризующая клиническую приверженность лечению, не зависит от возраста $\left(\chi^{2}=-0,19, p=0,18\right)$, пола $(m=1,0, p=0,32)$, пути выявления заболевания $(f=0,9, p=0,4)$, класса основного заболевания $(f=0,91, p=0,44)$, продолжительности заболевания $\left(\chi^{2}=-0,13, p=0,49\right)$, обострений $\left(\chi^{2}=-0,17, p=0,24\right)$, сопутствующих заболеваний $\left(\chi^{2}=0,006, p=0,9\right)$. 
5. Регулярность посещений выше ( $\varphi=5,3$ при $p<0,01)$ среди пациентов с двумя плановыми посещениями в год, чем среди с пациентов с тремя плановыми посещениями в год и более.

6. Зависимость сопутствующих заболеваний и обострений $\left(\chi^{2}=0,3, p=0,03\right)$, обострений и госпитализаций по сопутствующему заболеванию $\left(\chi^{2}=0,3, p=0,04\right)$, сопутствующих заболеваний и госпитализаций $\left(\chi^{2}=0,4, p=0,006\right)$, превышение частоты госпитализаций по сопутствующим заболеваниям над числом госпитализаций по основному заболеванию ( $m=-3,3, p=0,002)$ свидетельствует о том, что не уделяется достаточно внимания сопутствующим заболеваниям при диспансерном наблюдении.

7. До наступления инвалидности пациентам чаще на оказывается специализированная помощь: лица без инвалидности госпитализировались по сопутствующим заболеваниям чаще, чем инвалиды ( $m=2,5$, $p=0,02)$, хотя зависимости от наличия инвалидности, обострений, вызовов скорой и неотложной медицинской помощи не установлено $(p>0,05)$.

\section{БИБЛИОГРАФИЧЕСКИЙ СПИСОК}

1. Гадаборшев М.И., Левкович М.М., Рудлиикая Н.В. Организация оценки эффективности первичной медицинской помощи. М.: ИНФРА-М 2013; 424.

2. Гасанова А.3. Медико-организационные и социально-экономические аспекты диспансеризации работающего городского населения: автореф. дис. ... канд. мед. наук. М. 2012; 26.

3. Диспансерное наблюдение больных хроническими неинфекционными заболеваниями и пациентов с высоким риском их развития: метод. рекомендации. Под ред.
С.А. Бойцова и А.Г. Чучалина. М.: РОПИЗ 2014; 112.

4. Королева T.A. Научное обоснование совершенствования поликлинического диспансерного учета хронических неинфекционных заболеваний у лиц трудоспособного возраста: автореф. дис. ... канд. мед. наук. М. 2010; 24.

5. Кочубей А.В., Ластовецкий А.Г., Цветкова Е.А. Особенности нормативного правового регулирования организации профилактической помощи при реализации программ государственных гарантий оказания бесплатной медицинской помощи. Вестник Росздравнадзора 2015; 3: 64-66.

6. Овод А.И., Солянина В.А. Результаты исследования обращаемости женщин трудоспособного возраста за профилактической помощью. Возраст-ассоциированные и гендерные особенности здоровья и болезни: сб. материалов междунар. науч.-практ. конф. Курский гос. мед. ун-т. Курск 2016; 244-253.

7. Стародубов В.И., Калининская А.А., Сон И.М., Щепин В.О. Первичная медикосанитарная помощь: механизмы совершенствования. Вена: East West Association for Advanced Studies and Higher Education $\mathrm{GmbH}$ 2016; 118.

8. Lim AJ., Vervloet M., van Dijk L., Smit E.G., Van Weert J.M. Effects of health interventions on medication adherence: a systematic review of the literature. J Med Internet Res 2011; 13(4), available at: https://www.ncbi.nlm.nih.gov/ pmc/articles/PMC3278089

9. Viswanathan M., Golin C., Jones C., Ashok M., Blalock S., Wines $R$. et al. Interventions to improve adherence to self-administered medications for chronic diseases in the United States: a systematic review. Ann Intern Med 2012; 157 (11): 785-795.

Материал поступил в редакцию 20.12.2017 